Pacific Journal of Mathematics

MODULAR SUBLATTICES OF THE LATTICE OF VARIETIES
OF INVERSE SFMIGOUPS 


\title{
MODULAR SUBLATTICES OF THE LATTICE OF VARIETIES OF INVERSE SEMIGROUPS
}

\author{
N. R. REILLY
}

Kleiman used the variety $\mathscr{G} \mathscr{P}$ of all groups to define two endomorphisms $\varphi$ and $\varphi \mathscr{S}$ of the lattice $\mathscr{L}(\mathscr{F})$ of varieties of inverse semigroups as follows: $\varphi(\mathscr{V})=\mathscr{G} \mathscr{P} \vee \mathscr{V}$ and $\varphi_{\mathscr{O}},(\mathscr{V})=\mathscr{G} \mathscr{P} \wedge \mathscr{V}$. This introduced two congruences $\nu_{1}$ and $\nu_{2}$ on $\mathscr{L}(\mathscr{J})$ which have been very important in recent studies of $\mathscr{L}(\mathscr{J})$.

This paper is devoted to studying further properties of the $\nu_{1}$ and $\nu_{3}=\nu_{1} \cap \nu_{2}$ congruence classes.

The first main result establishes that each $\nu_{1}$-class is a complete modular sublattice of $\mathscr{L}(\mathscr{S})$, although, in some cases, the class may just consist of a single element.

It is not difficult to see that each $\nu_{3}$-class has a minimum member. On the other hand, it is shown that not all $\nu_{3}$-classes have maximum members. However, it is established that a large class of $\nu_{3}$-classes do have maximum members. If $\mathscr{U}$ is a variety satisfying an identity of the form $x^{n+1} t t^{-1} x^{-n-1}=$ $x^{n} t t^{-1} x^{-n}$ then the $\nu_{3}$-class containing $\mathscr{C}$ has a maximum member. The condition that a variety satisfies this identity is equivalent to a member of conditions, one being that every member of $\mathscr{V}$ is completely semisimple and such that $\mathscr{C}$ is a congruence.

The nature of the maximum element in these cases is very interesting. If $\mathscr{U}$ satisfies the above identity, then the fundamental inverse semigroups contained in $\mathscr{U}$ constitute a variety, $\mathscr{V}$ say. Letting $\mathscr{G}=\mathscr{G} \mathscr{P} \cap \mathscr{U}$, the maximum element in the $\nu_{3}$ class containing $\mathscr{U}$ is shown to be the Mal'cev product $\mathscr{G} \circ \mathscr{V}$ of the varieties $\mathscr{G}$ and $\mathscr{Y}$. It is shown that this is not valid in general. Other properties of the Mal'cev product are obtained.

1. Notation and terminology. We shall adopt the basic notation and terminology for semigroups from [2] while, for basic results in the theory of varieties of groups, the reader is referred to [10].

The variety of all inverse semigroups, (groups, abelian groups) will be denoted by $\mathscr{I}(\mathscr{G} \mathscr{P}, \mathscr{A} \mathscr{G} \mathscr{P})$ and the trivial variety by $\mathscr{T}$. Throughout the paper the term variety, if unqualified, will always mean a variety of inverse semigroups.

We will denote by $F_{X}\left(G_{X}\right)$ the free inverse semigroup (group) on a countable set $X$.

For any semigroup $S, \mathscr{C}(S)$ will denote the variety generated by $S$. For any variety $\mathscr{\mathscr { S }} F(\mathscr{V})$ will denote the relatively free inverse semigroup in $\mathscr{V}$ of countable rank and $\rho(\mathscr{V})$ will denote the 
verbal congruence on $F_{X}=F(\mathscr{F})$ defining the variety $\mathscr{V}$. In addition, $\mathscr{L}(\mathscr{Y})$ will denote the lattice of varieties contained in $\mathscr{Y}$.

For any group variety $\mathscr{G}$, we denote by $\mathscr{C}(\mathscr{G})$ the fully invariant subgroup of $G_{X}$ determining the variety $\mathscr{G}$. We shall also denote by $\mathscr{Q}(\mathscr{G})$ the class of varieties of such that oy $\cap \mathscr{G} \mathscr{P}=\mathscr{G}$. In particular, $\mathscr{Q}(\mathscr{T})$ denotes the class of those varieties that contain no nontrivial group.

For any inverse semigroup $S$, we will denote by $E(S)$ the semilattice of idempotents of $S$ and by $\mu_{S}$ the maximum idempotent separating congruence on $S$. For the basic properties of $\mu_{S}$, the reader is referred to [8].

For more extensive information on the background and context of this paper, the reader is referred particularly to the papers by Djadchenko [3], Kleiman [5], [6], and Reilly [12].

2. The modularity of $\nu_{3}$-classes. For any inverse semigroup $S$, we denote by $\Lambda(S)$ the lattice of congruences on $S$ and write

$$
\theta(S)=\left\{\left(\rho_{1}, \rho_{2}\right) \in \Lambda(S) \times \Lambda(S): \rho_{1} \cap E_{S} \times E_{S}=\rho_{2} \cap E_{S} \times E_{S}\right\} .
$$

From Reilly and Scheiblich [13], we have the following result.

Lemma 2.1. For any inverse semigroup $S$,

(1) $\theta(S)$ is a congruence on $\Lambda(S)$;

(2) each $\theta$-class is a complete modular sublattice of $\Lambda(S)$.

Let $\delta \subseteq F_{X} \times F_{X}$ and, for each $\left(u_{\lambda}, v_{\lambda}\right) \in \delta$ let $t_{\lambda}$ be an element of $X$ which does not appear in either $u_{\lambda}$ or $v_{\lambda}$. Then we write

$$
\bar{\delta}=\left\{\left(u_{\lambda} t_{\lambda} t_{\lambda}^{-1} u_{\lambda}^{-1}, v_{\lambda} t_{\lambda} t_{\lambda}^{-1} v_{\lambda}\right):\left(u_{\lambda}, v_{\lambda}\right) \in \delta\right\} .
$$

The following result is due to Kleiman [5].

Lemma 2.2. If $\delta$ is a basis for the identities of a variety of then $\bar{\delta}$ is a basis for the identities of $\mathscr{U} \vee \mathscr{G} \mathscr{P}$.

From this we immediately obtain the following corollary:

COROLlaRY 2.3. Let of be any variety. Then of $\vee \mathscr{G} \mathscr{P}=$ $\left\{S \in \mathscr{J}: S / \mu_{S} \in \mathscr{Y}\right\}$.

This leads us to the main result of this section. For varieties

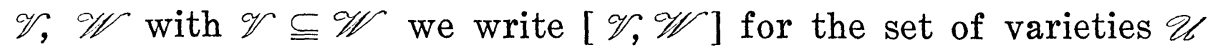
with $\mathscr{V} \subseteq \mathscr{Q} \subseteq \mathscr{Y}$. 
THEOREM 2.4. For any variety or of inverse semigroups, the interval $[\mathscr{Y}, \mathscr{Y} \vee \mathscr{G} \mathscr{P}]$ is a complete modular sublattice of $\mathscr{L}(\mathscr{F})$.

Proof. Let $\rho_{1}=\rho(\mathscr{V} \vee \mathscr{G} \mathscr{P})$ and $\rho_{2}=\rho(\mathscr{V})$. Then $F(\mathscr{V} \vee \mathscr{G} \mathscr{P})$ is just $F_{X} / \rho_{1}$ and $F(\mathscr{V})$ is just $F_{X} / \rho_{2}$. Let $\tau=\rho_{2} / \rho_{1}$. Then $\left(F_{X} / \rho_{1}\right) / \tau$ is isomorphic to $F_{X} / \rho_{2}$.

Since $F_{X} / \rho_{1} \in \mathscr{V} \vee \mathscr{G} \mathscr{P}$, it follows from Corollary 2.3 , that $\left(F_{X} / \rho_{1}\right) / \mu \in \mathscr{V}$; where $\mu$ is the maximum idempotent separating congruence on $F_{X} / \rho_{1}$. Suppose that we denote by $\rho_{3}$ the congruence on $F_{X}$ inducing $\mu$ on $F_{X} / \rho_{1}$, that is, such that $F_{X} / \rho_{3}$ is isomorphic to $\left(F_{X} / \rho_{1}\right) / \mu$. Then $F_{X} / \rho_{3} \in \mathscr{V}$ and so $\rho_{2} \subseteq \rho_{3}$. Hence $\tau=\rho_{2} / \rho_{1} \subseteq \rho_{3} / \rho_{1}=\mu$. Thus $\tau$ is an idempotent separating congruence on $F_{X} / \rho_{1}$. Hence $\left(\rho_{1} / \rho_{2}\right) \in \theta\left(F_{X}\right)$.

Now let $\mathscr{W} \in[\mathscr{Y}, \mathscr{P} \vee \mathscr{G} \mathscr{P}]$ and $\rho_{4}=\rho(\mathscr{W})$. Then $\rho_{1} \cong \rho_{4} \cong \rho_{2}$. Hence, $\rho_{1}, \rho_{2}$ and $\rho_{4}$ are all $\theta=\theta\left(F_{X}\right)$ equivalent.

Let us write $\mathscr{L}(\mathscr{Y}, \mathscr{V} \vee \mathscr{G} \mathscr{P})=[\mathscr{\mathscr { T }}, \mathscr{V} \vee \mathscr{G} \mathscr{P}]$ and $\mathscr{L}^{\prime}(\mathscr{Y}, \mathscr{V} \vee$ $\mathscr{G} \mathscr{P})$ for the lattice of fully invariant congruences of the form $\rho(\mathscr{W}), \mathscr{W} \in[\mathscr{Y}, \mathscr{V} \vee \mathscr{G} \mathscr{P}]$. Then $\mathscr{L}^{\prime}(\mathscr{V}, \mathscr{V} \vee \mathscr{G} \mathscr{P})$ is the set of all fully invariant congruences between (and including) $\rho(\mathscr{V})$ and $\rho(\mathscr{V} \vee \mathscr{G} \mathscr{P})$ and is anti-isomorphic to $\mathscr{L}(\mathscr{Y}, \mathscr{V} \vee \mathscr{G} \mathscr{P})$.

From the above, we see that $\mathscr{L}^{\prime}(\mathscr{V}, \mathscr{V} \vee \mathscr{G} \mathscr{P})$ is contained in a single $\theta$-class $A$, say, which, by Lemma 2.1 , is a modular sublattice of $\Lambda\left(F_{X}\right)$. Since $\mathscr{L}^{\prime}(\mathscr{V}, \mathscr{V} \vee \mathscr{G} \mathscr{P})$ is a sublattice of $\Lambda\left(F_{X}\right)$ and so of $A$, it follows that $\mathscr{L}^{\prime}(\mathscr{Y}, \mathscr{Y} \vee \mathscr{G} \mathscr{P})$ must be a modular lattice. Hence, $\mathscr{L}(\mathscr{F}, \mathscr{V} \vee \mathscr{G} \mathscr{P})$ is also modular, as required.

Since $\mathscr{L}(\mathscr{I})$ is a complete, it follows that $[\mathscr{V}, \mathscr{V} \vee \mathscr{G} \mathscr{P}]$ is a complete sublattice.

Let two mappings $\varphi^{\vee}$ and $\varphi^{\wedge}$ be defined on $\mathscr{L}(\mathscr{F})$ as follows: $\varphi^{\vee}(\mathscr{V})=\mathscr{V} \vee \mathscr{G} \mathscr{P}, \quad \varphi^{\wedge}(\mathscr{V})=\mathscr{V} \cap \mathscr{G} \mathscr{P}, \quad$ for all $\mathscr{V} \in \mathscr{L}(\mathscr{F})$.

LEMma 2.5. Kleiman [5]. The mapping $\varphi^{\vee}$ is a homomorphism of $\mathscr{L}(\mathscr{S})$ onto the lattice of varieties containing $\mathscr{G} \mathscr{P}$ and $\varphi^{\wedge}$ is a homomorphism of $\mathscr{L}(\mathscr{I})$ onto $\mathscr{L}(\mathscr{G} \mathscr{P})$.

This leads to certain useful partitions of $\mathscr{L}(\mathscr{J})$. Let $\nu_{1}\left(\nu_{2}\right)$ be the congruence on $\mathscr{L}(\mathscr{I})$ induced by $\mathscr{\varphi}^{\vee}\left(\mathscr{\varphi}^{\wedge}\right)$ and $\nu_{3}=\nu_{1} \cap \nu_{2}$. Thus,

$$
\begin{aligned}
& (\mathscr{Y}, \mathscr{W}) \in \nu_{1} \text { if and only if } \mathscr{Y} \vee \mathscr{G} \mathscr{P}=\mathscr{W} \vee \mathscr{G} \mathscr{P}, \\
& (\mathscr{Y}, \mathscr{W}) \in \nu_{2} \text { if and only if } \mathscr{V} \cap \mathscr{G} \mathscr{P}=\mathscr{W} \cap \mathscr{G} \mathscr{P} \text {. }
\end{aligned}
$$

Of course, for any group variety $\mathscr{G}$, the $\nu_{2}$-class containing $\mathscr{G}$ is just $\mathscr{Q}(\mathscr{G})$.

Recall [8] that an inverse semigroup is said to be fundamental 
if it has no nontrivial idempotent separating congruences or, equivalently, no nontrivial congruences contained in Green's relation $\mathscr{H}$. Such semigroups have also been called antigroups ([3] and elsewhere). We shall denote the class of the such inverse semigroups by $A \mathscr{G} \mathscr{P}$. It should be noted that this class is not itself a variety although it does have a role to play.

Proposition 2.6. Kleiman [5]. Let $\mathscr{Y}, \mathscr{W} \in \mathscr{L}(\mathscr{I})$. Then $\mathscr{P} \vee \mathscr{G} \mathscr{P}=\mathscr{W} \vee \mathscr{G} \mathscr{P}$ if and only if $\mathscr{V} \cap A \mathscr{G} \mathscr{P}=\mathscr{W} \cap A \mathscr{G} \mathscr{P}$.

This result provides us with a further description of $\nu_{1}$. Thus, for $\mathscr{Y}, \mathscr{W} \in \mathscr{L}(\mathscr{J})$,

$(\mathscr{Y}, \mathscr{W}) \in \nu_{1}$ if and only if $\mathscr{Y} \cap A \mathscr{G} \mathscr{P}=\mathscr{W} \cap A \mathscr{G} \mathscr{P}$.

Let us now focus our attention on a single $\nu_{1}$-class $\mathscr{N}$. Let $\mathscr{V} \in \mathscr{N}$. Then clearly $\mathscr{A}_{1}=\mathscr{V} \vee \mathscr{G} \mathscr{P}$ is the maximum element of $\mathscr{N}$. On the other hand, by Proposition $2.6 \mathscr{M}_{1} \cap A \mathscr{G} \mathscr{P} \cong \mathscr{V}$, for all $\mathscr{V} \in \mathscr{N}$. Hence, $\mathscr{L}_{0}=\mathscr{Y}\left(\mathscr{M}_{1} \cap A \mathscr{G} \mathscr{P}\right)$ is a minimum element of $\mathscr{N}$ and $\mathscr{A}_{1}=\mathscr{L}_{0} \vee \mathscr{G} \mathscr{P}$. Combining this with Theorem 2.4 we have the following result.

THeOREM 2.7. Let $\mathscr{N}$ be a $\nu_{1}$-class of $\mathscr{L}(\mathscr{F})$. Then $\mathscr{N}$ has both a minimum element, $\mathscr{L}_{0}$, and a maximum element, $\mathscr{H}_{1}=\mathscr{C}_{0} \mathrm{~V}$ $\mathscr{G} \mathscr{P}$. Moreover, $\mathscr{N}$ is a complete modular sublattice of $\mathscr{L}(\mathscr{I})$.

Since the minimum element of any $\nu_{1}$-class is generated by its fundamental elements and, conversely, any variety generated by its fundamental members is the minimum in its $\nu_{1}$-class, we say that any such variety is fundamental.

For any fundamental variety $\mathscr{F}$, let $\varphi^{\mathscr{N}}: \mathscr{L}(\mathscr{G} \mathscr{P}) \rightarrow[\mathscr{Y}, \mathscr{V} \vee$ $\mathscr{G} \mathscr{P}]$ be defined by

$$
\varphi^{\mathscr{V}}(\mathscr{G})=\mathscr{Y} \vee \mathscr{G}, \text { for all } \mathscr{G} \in \mathscr{L}(\mathscr{G} \mathscr{P}) \text {. }
$$

Then $\varphi^{\mathscr{V}}$ maps $\mathscr{L}(\mathscr{G} \mathscr{P})$ into $(\mathscr{V}) \nu_{1}$, the $\nu_{1}$-class containing $\mathscr{V}$.

It is interesting to note some of the situations that arise in this context.

Let $Y_{2}$ denote the two-element semilattice, $B_{2}$ denote the Brandt semigroup of rank two with trivial structure group and $B_{2}^{1}$ denote $B_{2}$ with an identity adjoined.

Then Djadchenko [3] has shown that for $\mathscr{V}$ equal to $\mathscr{V}\left(Y_{2}\right)$ or $\mathscr{V}\left(B_{2}\right), \varphi^{\mathscr{V}}$ is an isomorphism of $\mathscr{L}(\mathscr{G} \mathscr{P})$ onto $(\mathscr{V}) \nu_{1}$. Although $\mathscr{Y}\left(B_{2}^{1}\right)$ covers $\mathscr{Y}\left(B_{2}\right)$ in $\mathscr{L}(\mathscr{F})$, an example in [12], shows that $\varphi^{\mathscr{N}}$, where $\mathscr{V}=\mathscr{V}\left(B_{2}^{1}\right)$ does not map $\mathscr{L}(\mathscr{G} \mathscr{P})$ onto $\left(\mathscr{V}\left(B_{2}^{1}\right)\right) \nu_{1}$. However, 
$\varphi^{\prime}$ is still one-to-one.

At the other extreme, $(\mathscr{V}) \nu_{1}$ can be much smaller than $\mathscr{L}(\mathscr{G} \mathscr{P})$ and can even consist of a singleton, as the following example illustrates.

ExAmple. Let $T=\mathscr{C}^{\circ}\left(1 ; G_{X}, G_{X} ; \Delta\right)$ and $S=T \cup G_{X}$. Define a multiplication on $S$ by defining it to be the given multiplication within $T$ and $G_{X}$, such that the zero of $T$ is the zero of $S$ and such that, for any $g \in G_{X},(1 ; h, k) \in T$,

$$
\begin{aligned}
& g(1 ; h, k)=(1 ; g h, k) \\
& (1 ; h, k) g=\left(1 ; h, g^{-1} k\right) .
\end{aligned}
$$

It is a routine matter to check that $S$ is an inverse semigroup with respect to this multiplication. In fact, $S$ is a subsemigroup of the translational hull $\Omega(T)$ of $T$. Moreover, $S$ is fundamental. Hence, $\mathscr{T}(S)$ is fundamental. But clearly $G_{X} \in \mathscr{Y}(S)$. Therefore, $\mathscr{Y}(S) \vee$ $\mathscr{G} \mathscr{P}=\mathscr{Y}(S)$ and the $\nu_{1}$-class containing $\mathscr{Y}(S)$ contains only $\mathscr{Y}(S)$ itself.

Now $S$ satisfies the identity $x^{3} x^{-3}=x^{2} x^{-2}$ and so $x^{3} x^{-3}=x^{2} x^{-2}$ is an identity that is valid for $\mathscr{V}(S)$. Consequently, any element of $\mathscr{Y}(S)$ is completely semisimple (for details see [12]). Thus $\mathscr{V}(S)$ is certainly not the variety of all inverse semigroups, and, in some sense, is not far up the lattice $\mathscr{L}(\mathscr{I})$.

3. Maximum elements in $\nu_{3}$-classes. Since $\nu_{3}$-classes are sublattices of $\nu_{1}$-classes, it follows from Theorem 2.7, that each $\nu_{3}$-class is a modular sublattice of $\mathscr{L}(\mathscr{I})$. In this section we will show that each $\nu_{3}$-class has a minimum and, in some cases, also a maximum member.

Proposition 3.1. Let $V$ be a $\nu_{2}$-class. Let $\mathscr{W} \in V, \mathscr{G}=\mathscr{W} \cap$ $\mathscr{G} \mathscr{P}$ and $\mathscr{V}=\mathscr{Y}(\mathscr{W} \cap A \mathscr{G} \mathscr{P})$. Then $\mathscr{U}=\mathscr{G} \vee \mathscr{V}$ is the minimum member of $V$.

Proof. Since $\mathscr{W} \cap A \mathscr{G} \mathscr{P} \subseteq \mathscr{U}=\mathscr{G} \vee \mathscr{Y} \subseteq \mathscr{H}$, it follows that $\mathscr{U} \cap A \mathscr{G} \mathscr{P}^{\circ}=\mathscr{W}^{\circ} \cap A \mathscr{G} . \mathscr{P}$. Similarly,

$$
\mathscr{G} \subseteq \mathscr{U} \cap \mathscr{G} \mathscr{P} \subseteq \mathscr{W} \cap \mathscr{G} \mathscr{P}=\mathscr{G} .
$$

Hence $\mathscr{W} \cap \mathscr{G} \mathscr{P}=\mathscr{W} \cap \mathscr{G} \mathscr{P}$. Thus $(\mathscr{W}, \mathscr{W}) \in \nu_{3}$ and $\mathscr{\mathscr { C }} \cong \mathscr{W}$. Since $\mathscr{G}$ and $\mathscr{V}$ are independent of the choice of $\mathscr{W} \in V$, it follows that $\mathscr{C}$ is the minimum element of $V$.

Although we shall see in $\S 5$ that not all $\nu_{3}$-classes contain a maximum element, some do and we can identify the maximum element for a large family of $\nu_{3}$-classes. 
Let $\mathscr{C} \mathscr{S} \mathscr{H}$ denote the class of all completely semisimple inverse semigroups on which $\mathscr{H}$ is a congruence. This class is not itself a variety, however if we denote by $\mathscr{L}(\mathscr{C} \mathscr{S} \mathscr{C})$ the set of varieties contained in $\mathscr{C} \mathscr{S} \mathscr{Y}$, then $\mathscr{L}(\mathscr{C} \mathscr{S} \mathscr{H})$ is a sublattice of the lattice $\mathscr{L}(\mathscr{I})$ of all inverse semigroup varieties.

The class $\mathscr{C} \mathscr{S} \mathscr{C}$ was introduced in [12] where the following result was established.

THEOREM 3.2. Let $\mathscr{V}$ be a variety of inverse semigroups. Then the following statements are equivalent.

(1) For every $S \in \mathscr{C}, S$ is completely semisimple and $\mathscr{H}$ is a congruence: that is, $\mathscr{P} \in \mathscr{L}(\mathscr{C} \mathscr{S} \mathscr{H})$;

(2) $\mathscr{V}$ satisfies $x^{n+1} t t^{-1} x^{-n-1}=x^{n} t t^{-1} x^{-n}$, for some positive integer $n$.

(3) $\mathscr{V} \cap A \mathscr{G} \mathscr{P}$ satisfies $x^{n+1}=x^{n}$, for some positive integer $n$;

(4) $\mathscr{V} \cap A \mathscr{G} \mathscr{P}$ is a variety;

(5) $\mathscr{V} \cap A \mathscr{G} \mathscr{P} \in \mathscr{Q}(\mathscr{S})$.

Further insight into the nature of the conditions in Theorem 3.2 can be obtained from the next observation due to Djadchenko [3].

LEMMA 3.3. A variety or contains no nontrivial groups, that is $\mathscr{V} \in \mathbb{Q}(\mathscr{T})$, if and only if an identity of the form $x^{n+1}=x^{n}$ is valid in $\mathscr{V}$ for some positive integer $n$.

Note that, in the light of Lemma 3.3 and Proposition 2.6, conditions (3) and (4) of Theorem $3.2 \mathrm{imply}$ that $\mathscr{V}$ is $\nu_{1}$-equivalent to a variety in $\mathbb{Q}(\mathscr{S})$.

We shall show that $\nu_{3}$-classes containing varieties of the type identified in Theorem 3.2 or, equivalently, $\nu_{3}$-classes contained in $\nu_{1}$-classes that intersect nontrivially with $\mathscr{Q}(\mathscr{T})$ have maximum members. In order to identify the maximum member, we shall need the concept of the product of two classes of algebras introduced by Mal'cev [7].

Definition. If $\mathscr{C}$ and $\mathscr{V}$ are subclasses of a class $\mathscr{K}$ of algebras, then the product $\mathscr{U} \circ_{\mathscr{C}} \mathscr{V}$ is defined as consisting of the algebras $A$ from $\mathscr{K}$ such that for some congruence $\rho$ on $A, A / \rho \in \mathscr{V}$ and each $\rho$-class which is a subalgebra of $A$ is in $\mathscr{U}$.

If $\mathscr{U}, \mathscr{V}$ are varieties of groups and $\mathscr{K}$ is the variety of all groups, then $\mathscr{C} \circ_{\mathscr{X}} \mathscr{V}$ is just the standard product of group varieties, as studied in [10]. 
Although the product of two varieties of inverse semigroups has not been studied, in general, the work of Houghton [4] followed by that of Bales [1] has provided considerable information on the product of a variety of groups and a variety of inverse semigroups in the variety $\mathscr{F}$ of all inverse semigroups.

Since we shall only be considering products in the variety $\mathscr{A}$, we shall denote the product of two subvarieties $\mathscr{U}, \mathscr{V}$ of $\mathscr{F}$ simply by $\mathscr{C}_{6} \circ \mathscr{V}$, without any subscript.

We note in passing that the Mal'cev product of varieties of inverse semigroups is not always a variety of inverse semigroups. If we let $\mathscr{V}$ denote the variety of semilattices, then it follows from the construction of the free inverse semigroup due to Scheiblich [14] that $F_{X} \in \mathscr{Y} \circ \mathscr{G} \mathscr{P}$. Hence, any variety containing $\mathscr{Y} \circ \mathscr{G} \mathscr{P}$ must contain $\mathscr{F}$. However, any element $S$ of $\mathscr{Y} \circ \mathscr{G} \mathscr{P}$ must be $E$-unitary (that is, $a \in S$, $e$ and $e a \in E(S)$ implies that $a \in E(S)$ ) while not every element of $\mathscr{F}$ is $E$-unitary.

However, Bales [1] has shown that the product $\mathscr{C} \circ \mathscr{V}$ is a variety whenever $\mathscr{C}$ is a group variety.

As observed by Bales [1], any inverse semigroup identity $u\left(x_{1}, \cdots, x_{n}\right)=v\left(x_{1}, \cdots, x_{n}\right)$ is equivalent to the following two identities:

$$
\begin{aligned}
& u\left(x_{1}, \cdots, x_{n}\right) v\left(x_{1}, \cdots, x_{n}\right)^{-1} \\
& \quad=v\left(x_{1}, \cdots, x_{n}\right) v\left(x_{1}, \cdots, x_{n}\right)^{-1} \\
& u\left(x_{1}, \cdots, x_{n}\right)^{-1} u\left(x_{1}, \cdots, x_{n}\right) \\
& \quad=v\left(x_{1}, \cdots, x_{n}\right)^{-1} v\left(x_{1}, \cdots, x_{n}\right) .
\end{aligned}
$$

Thus, for any variety $\mathscr{C}$, there is a basis of identities of the form

$$
u\left(x_{1}, \cdots, x_{n}\right)=i\left(x_{1}, \cdots, x_{n}\right)
$$

where $i\left(x_{1}, \cdots, x_{n}\right)$ is an idempotent in $F_{X}$. For convenience, we abbreviate expressions of the form $u\left(x_{1}, \cdots, x_{n}\right)$ to $u(\bar{x})$.

If we write

$\operatorname{Idem}(\mathscr{V})=\left\{(u(\bar{x}), i(\bar{x})) \in F_{X} \times F_{X}: u(\bar{x})=i(\bar{x})\right.$ is an identity in $\mathscr{V}$ and $i(\bar{x})$ is an idempotent in $\left.F_{X}\right\}$,

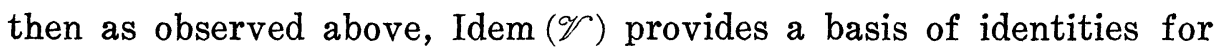
$\mathscr{T}$.

Combining Theorems 3.2 and 3.6 from Bales [1], we obtain the following important theorem.

THEOREM 3.4. Let $\mathscr{G}$ be a variety of groups and $\mathscr{V}$ a variety of inverse semigroups. Then $\mathscr{C} \circ \mathscr{C}$ is a variety. 
Moreover, the identities

$$
u\left(v_{1}\left(\bar{x}_{1}\right), \cdots, v_{n}\left(\bar{x}_{n}\right)\right)=i_{1}\left(\bar{x}_{1}\right) \cdots i_{n}\left(\bar{x}_{n}\right)
$$

for all $u(\bar{x}) \in U(\mathscr{G})$ and for all $\left(v_{j}\left(\bar{x}_{j}\right), i_{j}\left(\bar{x}_{j}\right)\right) \in \operatorname{Idem}(\mathscr{V})$, form a basis of identities for $\mathscr{G} \circ \mathscr{V}$.

We now combine Theorems 3.2 and 3.4 in order to show that a large class of $\nu_{3}$-classes contain maximum members which can be described by the Mal'cev product.

TheOREM 3.5. Let $\mathscr{U} \in \mathscr{L}(\mathscr{C} \mathscr{S} \mathscr{H})$. Let $\mathscr{G}=\mathscr{W} \cap \mathscr{G} \mathscr{P}$ and $\mathscr{V}=\mathscr{U} \cap A \mathscr{G} \mathscr{P}$. Then $\mathscr{G} \circ \mathscr{V}$ is the maximum member of the $\nu_{3}-$ class containing $\mathscr{U}$.

Proof. Let $\mathscr{W}$ be any element of the $\nu_{3}$-class containing $\mathscr{U}$. Then $\mathscr{W} \cap A \mathscr{G} \mathscr{P}=\mathscr{Q} \cap A \mathscr{G} \mathscr{P}=\mathscr{V}$. Therefore, for any $S \in \mathscr{W}$, $S / \mu_{S} \in \mathscr{V}$. Also, for any idempotent $e \in S$, since $\mathscr{W} \cap \mathscr{G} \mathscr{P}=\mathscr{U} \cap$ $\mathscr{G} \mathscr{P}=\mathscr{G}$, we have $e \mu_{S} \in \mathscr{G}$. Therefore, $\mu_{S}$ is a congruence on $S$ such that $S / \mu_{S} \in \mathscr{V}$ and each class of $\mu_{S}$ that is a subalgebra of $S$ belongs to $\mathscr{G}$. Hence, $S \in \mathscr{G} \circ \mathscr{Y}$. Therefore, $\mathscr{W} \subseteq \mathscr{G} \circ \mathscr{T}$.

To complete the theorem, therefore, it is only necessary to show that $(\mathscr{G} \circ \mathscr{V}, \mathscr{C}) \in \mathcal{\nu}_{3}$.

Since $\mathscr{C} \in \mathscr{L}(\mathscr{C} \mathscr{S} \mathscr{C})$, it follows from Theorem $3.2(3)$ that an identity of the form $x^{n+1}=x^{n}$ is valid in $\mathscr{V}=\mathscr{C} \cap A \mathscr{G} \mathscr{P}$, for some positive integer $n$. Therefore, if $v(x)=x^{n+1} x^{-n}$ then $\left(v(x), x^{n} x^{-n}\right) \in$ $\operatorname{Idem}(\mathscr{C})$.

Now let $G$ be any group in $\mathscr{G} \circ \mathscr{V}$ and let $u(\bar{x}) \in U(\mathscr{G})$. Then

$$
u\left(v\left(x_{1}\right), v\left(x_{2}\right), \cdots, v\left(x_{r}\right)\right)=x_{1}^{n} x_{1}^{-n} \cdots x_{r}^{n} x_{r}^{-n}
$$

is an identity that is valid in $\mathscr{G} \circ \mathscr{V}$. But, since $G$ is a group, for any $x \in G, v(x)=x$ and so the identity $u\left(x_{1}, \cdots, x_{r}\right)=1$ is valid in $G$ where 1 denotes the identity of $G$.

Since this is the case for all $u(\bar{x}) \in U(\mathscr{G})$, it follows that $G \in \mathscr{G}$. Hence, $(G \circ \mathscr{V}) \cap \mathscr{G} \mathscr{P} \subseteq \mathscr{G}$ and so, since it is clear that $\mathscr{G} \subseteq(\mathscr{G} \circ \mathscr{V}) \cap$ $\mathscr{G} \mathscr{P}$, it follows that $(\mathscr{G} \circ \mathscr{O}) \cap \mathscr{G} \mathscr{P}=\mathscr{G}$ and so $(\mathscr{G} \circ \mathscr{V}, \mathscr{U}) \in \nu_{2}$.

On the other hand, if $S \in \mathscr{G} \circ \mathscr{V}$ then there exists a congruence $\rho$ on $S$ such that $S / \rho \in \mathscr{V}$ and each $\rho$-class that is an inverse subsemigroup of $S$ lies in $\mathscr{G}$, that is, in particular, is a group. Hence, $\rho$ must be an idempotent separating congruence, and, by Corollary 2.3, we have $S \in \mathscr{G} \mathscr{P} \vee \mathscr{V}$. Hence, $\mathscr{G} \circ \mathscr{V} \cong \mathscr{G} \mathscr{P} \vee \mathscr{V}$ and so

$$
\mathscr{G} \mathscr{P} \vee \mathscr{Y} \subseteq \mathscr{G} \mathscr{P} \vee(\mathscr{G} \circ \mathscr{V}) \subseteq \mathscr{G}^{P} \vee \mathscr{Y} .
$$

Therefore, 


$$
\mathscr{G} \mathscr{P} \vee(\mathscr{G} \vee \mathscr{Y}) \subseteq \mathscr{G} \mathscr{P} \vee \mathscr{Y},
$$

and $(\mathscr{G} \circ \mathscr{Y}, \mathscr{U}) \in \nu_{1}$. Hence, $((\mathscr{G} \circ \mathscr{Y}), \mathscr{U}) \in \nu_{3}$, as required.

Since each $\nu_{3}$-class is determined by a fundamental variety and a group variety and vice versa, it will be convenient to denote the $\nu_{3}$-class determined by a group variety $\mathscr{G}$ and a fundamental variety $\mathscr{V}$ by $\nu_{3}(\mathscr{G}, \mathscr{V})$.

It is interesting to note that in the proof of the second half of Theorem 3.5, it is established that, for any variety $\mathscr{V}$ in which an identity of the form $x^{n+1}=x^{n}$ is valid (that is, for any $\mathscr{V} \in \mathbb{Q}(\mathscr{T})$ ) and for any group variety $\mathscr{G}, \mathscr{G} \circ \mathscr{V} \in \nu_{3}(\mathscr{G}, \mathscr{V})$. This enables us to restate Theorem 3.5 in terms of the group variety and fundamental variety determining a $\nu_{3}$-class.

CoRollary 3.6. Let $\mathscr{G}$ be a variety of groups and let $\mathscr{V} \epsilon$ $\mathscr{Q}(\mathscr{T})$. Then $\mathscr{G} \circ \mathscr{V}$ is the maximum element in $\nu_{3}(\mathscr{G}, \mathscr{Y})$.

4. Further results on the Mal'cev product. In this section we show that the Mal'cev product respects the lattice operations in $\mathscr{L}(\mathscr{C})$.

We recall (see [10]) that, for any subset $U$ of $G_{X}$, and, in particular, for any subgroup $U$ of $G_{X}$, and any group $G$, the verbal subgroup $U(G)$ of $G$ is the subgroup generated by the set $\{\alpha(u)$ : $u \in U$ and $\alpha$ is a homomorphism of $G_{X}$ into $\left.G\right\}$. A verbal subgroup is always fully invariant.

For a given variety of groups $\mathscr{C}$ corresponding to a fully invariant subgroup $U$ of $G_{X}, U=U\left(G_{X}\right)$. Furthermore, for any group $G, U(G)$ is the smallest normal subgroup of $G$ such that $G / U(G) \in \mathscr{C}$ and, in particular, $G$ belongs to $\mathscr{C}$ if and only if $U(G)=\{1\}$.

Lemma 4.1. Let of be a variety and $\left\{\mathscr{G}_{\lambda}: \lambda \in \Lambda\right\}$ be a family of varieties of groups. Then

$$
\left(\vee \mathscr{G}_{\lambda}\right) \circ \mathscr{Y}=\vee\left(\mathscr{C}_{\lambda} \circ \mathscr{Y}\right) \text { and }\left(\wedge \mathscr{C}_{\lambda}\right) \circ \mathscr{V}=\wedge\left(\mathscr{G}_{\lambda} \circ \mathscr{Y}\right) .
$$

Proof. It is clearly the case that $\left(\mathscr{C}_{\lambda} \circ \mathscr{V}\right) \subseteq\left(\vee \mathscr{C}_{\lambda}\right) \circ \mathscr{V}$ and so that $\vee\left(\mathscr{G}_{\lambda} \circ \mathscr{V}\right) \cong\left(\mathscr{G}_{\lambda}\right) \circ \mathscr{Y}$.

Let $S \in\left(\vee \mathscr{G}_{\lambda}\right) \circ \mathscr{T}$. Then there exists an idempotent separating congruence $\rho$ on $S$ such that $S / \rho \in \mathscr{V}$ and $N_{e}=e \rho \in \vee \mathscr{G}_{\lambda}$, for all $e \in E(S)$. For each $\lambda$, let $U^{\lambda}=U\left(\mathscr{C}_{\lambda}\right), U=U\left(\vee \mathscr{G}_{\lambda}\right)$ and $N_{e}^{\lambda}=U^{\lambda}(N \hat{e})$. Since $\left\{N_{e}: e \in E(S)\right\}$ is the kernel normal system for an idempotent separating congruence, $N=\cup\left\{N_{e}: e \in E(S)\right\}$ is, in particular, a semilattice of groups. It can then be verified quite routinely that $\left\{N_{e}^{\lambda}\right.$ : 
$e \in E(S)\}$ is a kernel normal system for each $\lambda$. Let the corresponding congruence, which is necessarily idempotent separating, be denoted by $\rho_{\lambda}$.

Now $\cap U^{\lambda}=U$. Hence, since $N_{e} \in \vee \mathscr{G}_{2}$, for each $e \in E(S)$, we must have $\cap N_{e}^{\lambda}$ equal to the trivial subgroup of $N_{e}$. Therefore, $\cap \rho_{\lambda}$ is the identity congruence. Now, for each $\lambda, \rho / \rho_{\lambda}$ is an idempotent separating congruence on $S / \rho_{\lambda}$ and $\left(S / \rho_{\lambda}\right) /\left(\rho / \rho_{\lambda}\right)$ is isomorphic to $S / \rho$ and so lies in $\mathscr{V}$. Let $e \rho_{\lambda}$ be any idempotent of $S / \rho_{\lambda}$. Then the $\rho / \rho_{\lambda}$-class containing $e \rho_{\lambda}$ is isomorphic to $N_{e} / N_{e}^{\lambda}$ and so, by the definition of $N_{e}^{\lambda}$, lies in $\mathscr{C}_{\lambda}$. Hence, for each $\lambda, S / \rho_{\lambda} \in$ $\mathscr{G}_{\lambda} \circ \mathscr{Y}$ and $\cap \rho_{\lambda}$ is the identity congruence. Therefore, $S \in \vee\left(\mathscr{G}_{\lambda} \circ \mathscr{V}\right)$ and the first half of the lemma is established.

With regard to the second assertion, it is again the case that the inclusion one way is trivial, namely, $\left(\wedge \mathscr{G}_{\lambda}\right) \circ \mathscr{V} \cong \wedge\left(\mathscr{G}_{\lambda} \circ \mathscr{V}\right)$.

Let $S \in \wedge\left(\mathscr{G}_{\lambda} \circ \mathscr{V}\right)$. Then $S \in \mathscr{G}_{\lambda} \circ \mathscr{V}$, for all $\lambda$. Hence, for each $\lambda$, there exists an idempotent separating congruence $\rho_{\lambda}$ on $S$ such that $e \rho_{\lambda} \in \mathscr{G}_{\lambda}$, for all $e \in E(S)$, and $S / \rho_{\lambda} \in \mathscr{V}$.

Let $\rho=\cap \rho_{\lambda}$. For each $e \in E(S)$, $e \rho=\cap e \rho_{\lambda} \in \wedge \mathscr{G}_{\lambda}$ while $S / \rho$, as a subdirect product of the $S / \rho_{\lambda}$, lies in $\mathscr{V}$. Hence, $S \in\left(\wedge \mathscr{G}_{\lambda}\right) \circ \mathscr{V}$ and the second part of the lemma is established.

From Corollary 3.3 of [1] and Corollary 2.3, we have the following result.

LEMmA 4.2. If $\mathscr{V}$ is a variety such that $\mathscr{G} \mathscr{P} \nsubseteq \mathscr{V}$, then the mapping $\mathscr{G} \rightarrow \mathscr{G} \circ \mathscr{V}$ is a one-to-one order isomorphism of $\mathscr{L}(\mathscr{G} \mathscr{P})$

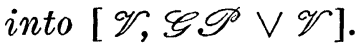

Combining Lemmas 4.1 and 4.2 we then have the following.

THEOREM 4.3. If $\mathscr{V}$ is a variety such that $\mathscr{G} \mathscr{P} \nsubseteq \mathscr{V}$ then the mapping $\mathscr{G} \rightarrow \mathscr{G} \circ \mathscr{V}$ is a complete lattice isomorphism of $\mathscr{L}(\mathscr{G} \mathscr{P})$ into [ $\mathscr{Y}, \mathscr{G} \mathscr{P} \vee \mathscr{V}]$.

5. A detailed study of a $\nu_{2}$-class. In this section it will be shown that there exist $\nu_{3}$-classes that have no maximum elements. Moreover, the class used to demonstrate this will contain a variety of the form $\mathscr{G} \circ \mathscr{F}$, where $\mathscr{G}$ is a group variety and $\mathscr{V}$ is a fundamental variety, thus showing, in addition, that such varieties are not always maximum in their classes.

We denote by $\mathscr{C}$ the bicyclic semigroup.

Let $G$ be a group, $\alpha$ an endomorphism of $G$, and $N$ the set of nonnegative integers. Then we denote by $B(G, \alpha)$ the set $N \times G \times N$ under the multiplication 


$$
(m ; g ; n)(p ; h ; q)=\left(m+p-r ; \alpha^{p-r}(g) \alpha^{n-r}(h) ; n+q-r\right),
$$

where $r=\min (n, p)$.

With respect to this multiplication $B(G, \alpha)$ is a bisimple inverse semigroup. For the basic properties of such semigroups the reader is referred to [9] and [11].

For any elements $x, y$ of an inverse semigroup $S$, we denote by $[x, y]$ the "commutator" element $x^{-1} y^{-1} x y$. We require some elementary facts about elements of this form.

Lemma 5.1. Let $S$ be an inverse semigroup, $G$ be a group and $\alpha$ an endomorphism of $G$.

(1) If either $x$ or $y$ belongs to $E(S)$ then so does $[x, y]$.

(2) For any $x, y \in \mathscr{C},[x, y] \in E(\mathscr{C})$.

(3) For any $x, y \in B(G, \alpha)$, $[x, y]$ belongs to a subgroup of $B(G, \alpha)$.

Proof. The observations can be verified by straightforward computations.

We recall the definition of the wreath product of two groups $G$ and $H$. We denote by $G^{H}$ the group of functions from $H$ to $G$ where the group operation is defined componentwise. Then, by the wreath product $G$ Wr $H$ of $G$ and $H$ we mean $H \times G^{H}$ with multiplication defined by

$$
(b, f)(c, g)=\left(b c, f^{c} g\right)
$$

where $f^{c}(y)=f\left(y c^{-1}\right)$, for all $y \in H$. With respect to this operation $G \mathrm{Wr} H$ is a group [10]. In particular, $(b, f)^{-1}=\left(b^{-1},\left(f^{d}\right)^{-1}\right)$, where $d=b^{-1}$.

For each positive integer $i$, let $G_{i}$ denote the product of $i$ copies of $Z \mathrm{Wr} Z$. We denote the identities of $Z \mathrm{Wr} Z$ and $G_{i}$ by 1 . For $k, b \in Z$ let $f_{k, b} \in Z^{Z}$ be defined as follows:

$$
f_{k, b}(z)=\left\{\begin{array}{lll}
b & \text { if } & z=k \\
0 & \text { if } & z \neq k
\end{array} .\right.
$$

Let $\alpha_{i}: G_{i} \rightarrow G_{i}$ be defined as follows: for all $F \in G_{i}$,

$$
\alpha_{i}(F)(n)=\left\{\begin{array}{lll}
1 & \text { if } & n=1 \\
F(n-1) & \text { if } & 1<n \leqq i
\end{array}\right.
$$

It is straightforward to verify that $\alpha_{i}$ is an endomorphism of $G_{i}$ such that $\alpha_{i}^{i}$ is the zero endomorphism and, for all $F \in G_{i}$,

$$
\alpha_{i}^{i-1}(F)(n)=\left\{\begin{array}{lll}
1 & \text { if } & 1 \leqq n<i \\
F(1) & \text { if } & n=i
\end{array}\right.
$$


Since the group operation in $Z$ is addition, we denote the group operation in $Z^{Z}$ by addition also.

Let $B_{i}=B\left(G_{i}, \alpha_{i}\right)$.

Since $G_{i}$ is a product of wreath products of the integers it follows that $G_{i} \in \mathscr{A} \mathscr{G} \mathscr{P} \circ \mathscr{A C \mathscr { P }}$, for all $i$. Hence, the identity

$$
[[x, y],[u, v]]^{2}=[[x, y],[u, v]]
$$

is valid in the group $\mathscr{H}$-classes of $B_{i}$.

Lемма 5.2. Let $e$ denote the product of the words of the form $w^{i} w^{-i} w^{-i} w^{i}$ where $w=x, y, u, v, x y, y x, u v, v u$. Then the identity

$$
[[e x e, e y e],[e u e, e v e]]^{2}=[[e x e, e y e],[e u e, e v e]]
$$

is valid in $B_{i}$.

Proof. We omit the details of the proof which consists of straightforward computations.

CoRollary 5.3. $\mathscr{Y}\left(B_{i}\right) \in \nu_{3}(. \mathscr{A} \mathscr{G} \mathscr{P} \circ \mathscr{A} \mathscr{G} \mathscr{P}, \mathscr{Y}(\mathscr{C}))$.

Proof. Since $B_{i} / \mu_{B_{i}}$ is isomorphic to $\mathscr{C}$, we have from Corollary 2.3 that $B_{i} \in[\mathscr{V}(\mathscr{C}), \mathscr{V}(\mathscr{C}) \vee \mathscr{G} \mathscr{P}] . \quad$ By Lemma $5.2,\left(^{*}\right)$ is valid in $\mathscr{V}\left(B_{i}\right)$ and so the identity

$$
[[x, y],[u, v]]^{2}=[[x, y],[u, v]]
$$

holds in $\mathscr{V}\left(B_{i}\right) \cap \mathscr{G} \mathscr{P}$. Thus $\mathscr{V}\left(B_{i}\right) \cap \mathscr{G} \mathscr{P} \subset \mathscr{A} \mathscr{G} \mathscr{P} \circ \mathscr{A} \mathscr{G} \mathscr{P}$. Since $Z W r Z \in \mathscr{V}\left(B_{i}\right)$ it follows that $\mathscr{Y}\left(B_{i}\right) \cap \mathscr{G} \mathscr{P}=\mathscr{A} \mathscr{C} \mathscr{P} \circ \mathscr{A} \mathscr{G} \mathscr{P}$.

A simple argument using the fact that, for any group variety $\mathscr{G}$ and any inverse semigroup variety $\mathscr{V}, \mathscr{G} \circ \mathscr{V} \cap \mathscr{G} \mathscr{P}=\mathscr{G} \circ(\mathscr{V} \cap \mathscr{G} \mathscr{P})$ will now establish that the varieties $\mathscr{C}\left(B_{i}\right)$ also contain $\mathscr{A} \mathscr{G} \mathscr{P}$ 。 $\mathscr{V}(\mathscr{C})$. Hence, we have the following proposition.

Proposition 5.4. For any positive integer $i$,

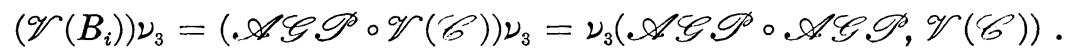

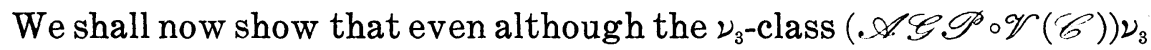
contains a Mal'cev product of varieties, it has no maximum member.

We proceed by contradiction. Suppose that there is a maximum member $\mathscr{V}$. Then $\mathscr{V}\left(B_{i}\right) \subseteq \mathscr{V}$, for all $i$, and so $B_{i} \in \mathscr{V}$, for all $i$. Also, $\mathscr{V} \wedge \mathscr{G} \mathscr{P}=\mathscr{A} \mathscr{G} \mathscr{P}^{\circ} \circ \mathscr{A} \mathscr{G} \mathscr{P}^{\circ}$ and so must satisfy the identity

$$
[[x, y],[u, v]]^{2}=[[x, y],[u, v]] .
$$

Let $S$ denote the inverse subsemigroup of the product $\Pi\left\{B_{i} ; i=\right.$ 
$1,2,3, \cdots\}$ consisting of those elements $F$ such that there exist some fixed integers $m$ and $n$ (dependent on $F$ ) such that $F(i)=\left(m ; A_{i} ; n\right)$, for some $A_{i} \in G_{i}$, for all $i=1,2,3, \cdots$.

Let $X, Y, U, V$ be defined as follows: for all $i=1,2,3, \cdots$, $X(i)=U(i)=\left(1 ; A_{i} ; 0\right), \quad Y(i)=\left(1 ; B_{i} ; 1\right), \quad V(i)=\left(1 ; C_{i} ; 1\right)$ where, for $1 \leqq n \leqq i$,

$$
\begin{aligned}
& A_{i}(n)=1, \quad \text { the identity of } Z \mathrm{Wr} Z, \\
& B_{i}(n)=\left(1, f_{0,1}\right), \quad C_{i}(n)=\left(2, f_{0,1}\right) .
\end{aligned}
$$

It can now be checked that there is no idempotent $e$ in $S$ for which $e[[X, Y],[U, V]]$ is an idempotent. Therefore $S / \sigma$, where $\sigma$ is the minimum group congruence on $S$, does not satisfy $\left(^{* *}\right)$. Therefore, $S \notin \mathscr{V}$ and we have the following result.

THEOREM 5.5. The $\nu_{3}$-class containing $\mathscr{A} \mathscr{G} \mathscr{P} \circ \mathscr{V}(\mathscr{C})$ has no maximum member.

\section{REFERENCES}

1. J. L. Bales, On product varieties of inverse semigroups, J. Australian Math. Soc., (1979), 107-119.

2. A. H. Clifford and G. B. Preston, The algebraic theory of semigroups, Amer. Math. Soc., Math. Surveys, 7 (1961 and 1967), Providence.

3. G. G. Djadchenko, On identities in monogenic inverse semigroups, Aigebra and Theory of Numbers, Nalčik, Issue 2 (1977), 57-77 (Russian).

4. C. H. Houghton, Embedding inverse semigroups in wreath products, Glasgow Math. J., 17 (1976), 77-82.

5. E. I. Kleiman, On the lattice of varieties of inverse semigroups, Izvestia Vyssih Učebnih Zavedenii. Matematika, No. 7 (1976), 106-109.

$6 . \quad$ On bases of identities of Brandt semigroups, Semigroup Forum, 13 (1977), 209-218.

7. A.I. Mal'cev, Multiplication of classes of algebraic systems, Sibir. Mat. Z., (1967), 346-365 (Russian).

8. W. D. Munn, Fundamental inverse semigroups, Quarterly J. Math. Oxford, (2), 21 (1970), 157-170.

9. W. D. Munn and N. R. Reilly, Congruence on a bisimple $\omega$-semigroup, Proc. Glasgow Math. Assoc., 7 (1966), 184-192.

10. H. Newmann, Varieties of groups, Ergebnisse d. Math. u. i Grenzgebiete, Springer. Verlag, (1967), Berlin.

11. N. R. Reilly, Bisimple w-semigroups, Proc. Glasgaw Math. Assoc., 7 (1966), 160-169.

12. - Varieties of completely semisimple inverse semigroups, (to appear).

13. N. R. Reilly and H.E. Scheiblich, Congruences on regular semigroups, Pacific J. Math., 23 (1967), 349-360.

14. H. E. Scheiblich, Free inverse semigroups, Proc. Amer. Math. Soc., 38 (1973), 1-7. A 4044 .

Received November 30, 1978. This research was supported, in part, by NRC Grant

Simon Fraser UNIVERSITY

BURNABY

British Columbia, Canada 



\section{PACIFIC JOURNAL OF MATHEMATICS}

\section{EDITORS}

DONALD BABBITT (Managing Editor)

University of California

Los Angeles, CA 90024

HUGo RossI

University of Utah

Salt Lake City, UT 84112

C. C. MOORE and ANDREW OGG

University of California

Berkeley, CA 94720
J. DugundJI

Department of Mathematics

University of Southern California

Los Angeles, CA 90007

R. FinN and J. Milgram

Stanford University

Stanford, CA 94305

ASSOCIATE EDITORS
E. F. BECKENBACH
B. H. NeUmanN
F. WOLF
K. YoSHIDA

\section{SUPPORTING INSTITUTIONS}

UNIVERSITY OF BRITISH COLUMBIA

CALIFORNIA INSTITUTE OF TECHNOLOGY

UNIVERSITY OF CALIFORNIA

MONTANA STATE UNIVERSITY

UNIVERSITY OF NEVADA, RENO

NEW MEXICO STATE UNIVERSITY

OREGON STATE UNIVERSITY

UNIVERSITY OF OREGON
UNIVERSITY OF SOUTHERN CALIFORNIA

STANFORD UNIVERSITY

UNIVERSITY OF HAWAII

UNIVERSITY OF TOKYO

UNIVERSITY OF UTAH

WASHINGTON STATE UNIVERSITY

UNIVERSITY OF WASHINGTON

The Supporting Institutions listed above contribute to the cost of publication of this Journal, but they are not owners or publishers and have no responsibility for its content or policies.

Mathematical papers intended for publication in the Pacific Journal of Mathematics should be in typed form or offset-reproduced, (not dittoed), double spaced with large margins. Please do not use built up fractions in the text of the manuscript. However, you may use them in the displayed equations. Underline Greek letters in red, German in green, and script in blue. The first paragraph or two must be capable of being used separately as a synopsis of the entire paper. Please propose a heading for the odd numbered pages of less than 35 characters. Manuscripts, in triplicate, may be sent to any one of the editors. Please classify according to the scheme of Math. Reviews, Index to Vol. 39. Supply name and address of author to whom proofs should be sent. All other communications should be addressed to the managing editor, or Elaine Barth, University of California, Los Angeles, California, 90024.

50 reprints to each author are provided free for each article, only if page charges have been substantially paid. Additional copies may be obtained at cost in multiples of 50 .

The Pacific Journal of Mathematics is issued monthly as of January 1966. Regular subscription rate: $\$ 84.00$ a year (6 Vols., 12 issues). Special rato: $\$ 42.00$ a year to individual members of supporting institutions.

Subscriptions, orders for numbers issued in the last three calendar years, and changes of address shoud be sent to Pacific Journal of Mathematics, P.O. Box 969, Carmel Valley, CA 93924, U.S.A Old back numbers obtainable from Kraus Periodicals Co., Route 100, Millwood, NY 10546.

PUBLISHED BY PACIFIC JOURNAL OF MATHEMATICS, A NON-PROFIT CORPORATION

Printed at Kokusai Bunken Insatsusha (International Academic Printing Co., Ltd.). 8-8, 3-chome, Takadanobaba, Shinjuku-ku, Tokyo 160, Japan.

Copyright (C) 1980 by Pacific Jounal of Mathematics Manufactured and first issued in Japan 


\section{Pacific Journal of Mathematics \\ Vol. 89, No. $2 \quad$ June, 1980}

Frank Hayne Beatrous, Jr. and R. Michael Range, On holomorphic

approximation in weakly pseudoconvex domains................. 249

Lawrence Victor Berman, Quadratic forms and power series fields ...... 257

John Bligh Conway and Wacław Szymański, Singly generated antisymmetric operator algebras ....................... 269

Patrick C. Endicott and J. Wolfgang Smith, A homology spectral sequence for submersions . . . .................................

Sushil Jajodia, Homotopy classification of lens spaces for one-relator groups with torsion ................................ 301

Herbert Meyer Kamowitz, Compact endomorphisms of Banach

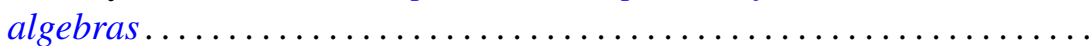

Keith Milo Kendig, Moiré phenomena in algebraic geometry: polynomial

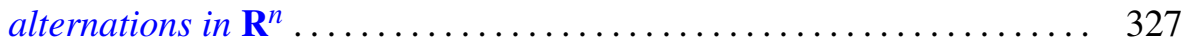

Cecelia Laurie, Invariant subspace lattices and compact operators....... 351

Ronald Leslie Lipsman, Restrictions of principal series to a real form . . . . . 367

Douglas C. McMahon and Louis Jack Nachman, An intrinsic

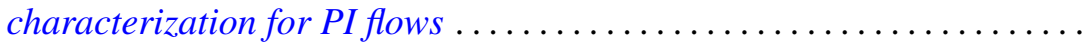

Norman R. Reilly, Modular sublattices of the lattice of varieties of inverse semigroups .................................... 405

Jeffrey Arthur Rosoff, Effective divisor classes and blowings-up of $\mathbf{P}^{2}$ 419

Zalman Rubinstein, Solution of the middle coefficient problem for certain

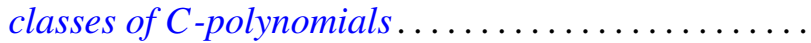

Alladi Sitaram, An analogue of the Wiener-Tauberian theorem for spherical transforms on semisimple Lie groups ................

Hal Leslie Smith, A note on disconjugacy for second order systems ...

J. Wolfgang Smith, Fiber homology and orientability of maps ...

Audrey Anne Terras, Integral formulas and integral tests for series of positive matrices. 